$\downarrow$

\section{ON THE EFFECTS OF SCARLET FEVER UPON THE EAR.}

By GEORGE PILCHER, Esq., formerly President of the Medical Society of London, etc.

[Read before the Medical Society of London, Dee. 16th, 1854.]

Wrut is the condition or nature, or what are the conditions or circumstances of the disease, which render scarlet ferer specific or peculiar, may be well worth the inquiry of a scientific society. The term specific, as applied to it, appears to be justified by the facts, that, as far as we know, it originates from a specific or peculiar cause, though in many instances we are unable to trace it to its cause, and in others it certainly appears to arise sporadically ; that it runs through a peculiar course, greatly modified by attendant circumstances and conditions, both extraneous and inherent; and that it has peculiar results, as we have a right, I think, to presume, though, in the present state of our knowledge of pathology, and of minute morbid anatomy in particular, we cannot distinguish some of these consequences from those of other febrile diseases. Scarlet fever is essentially a disease of the dermoid structures, both internal and external; but whether the specific affection resides in the general disease or the fever, producing its local evidences in the cutaneous and mucous structures; or in the local inflammation, of which the fever is merely sympathetic, - can be better decided by many other fellows of the society than by me. It would form an interesting inquiry, also, whether the specific character of the disease extended from its early dermoid location to the contiguous structures, or whether that extension was mere common inflammation. Again, it may be inquired if any one part of the dermoid tissue is more obnoxious to the disease than another; and some facts will be detailed in this paper, which may possibly lead to the supposition that the ear, being more frequently affected, and more fatally disorganised than other external organs, possesses some peculiarity of structure which more readily associates it with the peculiarity of scarlatina. That this organ of sense is especially obnoxious to the effects of this disease, I entertain no doubt; but that it is consequent upon what may fairly be termed peculiarity of structure or idiosyncrasy, I have grave misgivings; unless indeed its situation, and its various structures necessary for the performance of its functions, are regarded as peculiar. The mechanical portions of the ear, essentially developments of the dermoid textures, both cutaneous and mucous-prolongations (if such a word be justifiable in medical science) of those portions of the structures which are early and usually more severely affected by the exanthemata, facilitate the spread of the eruptive inflammation down to the membrana tympani on the one side, and along the Eustachian tube to the tympanal cavity on the other, thus meeting on the membrane; this, and the high degree of organization, as well as delicacy of the mucous lining, are circumstances sufficient, in my opinion, to account for the frequent disorganisations, which arise from scarlet-fever, without presupposing that any of the structures entering into the formation of the ear unenviably enjoy a scarlatinous idiosyncrasy beyond all other dermoid tissues. The disorganising effects of scarlet fever upon the ear are unhappily too notorious to be gainsaid; and equally unhappy is it that the attention of medical practitioners is insufficiently, and in many instances not at all, directed to this distressing result of the disease, and especially at the period when medical treatment might ararest or modify its otherwise fatal progress. It is an excuse sometimes that the condition of the patient is so desperate that, in the struggle to save life, the minor effects of the disease are for the time disregarded, or even orerlooked; in the former case, it being imagined time enough to attend to the ear when the patient becomes convalescent, or, at all crents, is out of danger to life. This is a fatal error, against which I would gladly raise $\mathrm{my}$ voice to its very highest pitch. I well know, in most cases of such extremity, that much in the way of local treatment is not to be effected; but I know likewise that, in all cases, many aggravating conditions attending the sad disease may be alleviated; and that in many its progress may be arrested, its severity diminished. Let us not forget that death is not alwrys the most unhappy consequence of disease; and that life in the young and innocent, dependent upon its own exertions for its future welfare, perhaps its very existence, may be over-dearly purchased by a total deafness, insuring a consequent dumbness. I wish not to be misunderstood to imply that it is not invariably the practitioner's duty to use his utmost exertions to save life under all and every circumstance, leaving the consequences of that life to Infinite Wisdom. But if it were possible to happen that the necessary local treatment for the ear were antagonistic to the general treatment, in such extreme cases some hazard to life is justified, nay, called for, in the attempt to ward off such a dire calamity as the destruction of an organ of sense; more particularly so if both were involved. If possible, a still mure important circumstance is not infrequently overlooked in the fuct that this secondary disease in the ear may now be the essential affection under which the patient labours, and which is eventually to destroy him. We all recognise how rarely an original affection terminates in fatality; but that death ensues upon the secondary or tertiary, or still more subsequent effect; and scarlet fever does not form an exception to that general law.

In briefly noticing the principal effects of scarlet fever upon the ear, it will be convenient to trace them from without inwards, rather than according to the frequency with which they occur.

The auricle is essentially liable to be affected with all eruptive diseases, in consequence of its exposure, of injurious pressure occasioned by lying upon it, and of the great vascularity of its integument. The inflammation, extending to the cartilage, not infrequently produces permanent hypertrophy, and occasionally other consequences. If the thickening occupying the concha should encroach upon the opening into the auditory meatus, it becomes a troublesome cause of deafness, very difficult to remove. The auricle is of course the subject of the various chronic effects of acute specific intlammation which are occasionally observed in other parts of the integument; and not infrequently gangrene results in the acute form of scarlet fever.

The eruptive disease spreads along the external canal, and over the surface of the membrana tympani; most probably, in the generality of cases, except they be of the mildest character, giving rise to the uncasiness and heat of the part, and slight deafness, so constantly occurring, and yet so frequently unattended to. This condition is followed by the customary desquamation of the cuticle from the canal and surface of the membrane, and frequently requires the syringe for its complete removal. In more serere cases, the lining membrane becomcs inflauned, which may extend to' the cellular tissue, to the fibrous and cartilaginous structures beneath, and likewise to the fibrous layers of the membrana tympani, accompanied by corresponding symptoms of aggravated pain and intolerance of sound, and endangering permanent thickening of the membrana tympani in particular, or ulceration with otorrhcea, and its sad inconveniencies. Again, an early desquamation of the cuticle may occur, followed by a discharge of seromucus, and afterwards of muco-pus, or very often of a matter apparently combined of all three, sero-muco-pus, being flaky, as if bits of epithelium were floating in it. This discharge probably proceeds from the inflamed ceruminous follicles; yet, from the after effects, there is reason to believe that the entire length of the tube furnishes it. The same degree of inflammation affects the membrans tympani, which is especially prone to effusion of fibrin between or in its layers; and hence it furnishes a frequent cause of permanent thickening of that membrane, and corresponding loss of vibratibility, and hence correspending 
danfmess. In neglected cases of this kind, the cuticle is not reproduced; the dermis becomes thick, with spongy granuhtions; and an otorrhoes, attended with more or less deafnese, and occasional bleeding, is established. Now and then, a fungoid polypus springs up from the diseased skin and cellular membrane, very vascular, easily bleeding, frequently alarming to the patient and friends, but, when confined to the lining membrane, generally very manageable. Ulceration may extend to the cartilaginous and to the fibro-celluiar parietes of the cannl, and even through the canal, and open externally, either in front of the tragus, or behind the concha, in front of the mastoid process: the latter situation is the more frequent. It generally happens that a fungoid growth projects from the ulcer into the meatus. These cases are always difficult of cure, and sometimes rery much so; their progress and extension being uncertain, and so much influenced by and depending upon the general health. Abscesses external to the ear, in the glands and cellular tissue around the meatus, and ulcerating into the canal, are another source of discharge from the outer tube, and of fungus projecting into it, independent of disease of the middle car; and which is often attended with great inconvenience and trouble, endangering extension onwards to the tympanum, and hence, it may be, to the internal ear. Intractable fistulous ulcers, of considerable length, running under the deep cervical fascia in various directions, and caries of the processus auditivus, are not very infrequent consequences of this state of the external ear.

We find, however, that scarlet fever makes its saddest, its most direful ravages, in the carity of the tympanum; and thence extends its disorganising fatal effects in erery direction; generally, fortunately, outwards through the membrana tympani, backwards to the mastoidal cells, inwards to the cochlea, through the bony walls to the brain whether it be upwards to the cerebrum, or through the pnsterior wall to the cerebellum. I must, however, state, in limine, that, according to my experience, scarlet fever does not kill during its own progress by abscess in the brain, as an extension of disease from the ear, so frequently as the constant occurrence of destructive scarlatina in the middle car would lead us to expect. But then, again, I must in honesty modify this statement by expressing my belief that many cases of fatal scarlet ferer are deaths from abscess or other disease of the brain, extensions from the tympanum, but unrecognised during life by the practitioner. I know this fact has occurred in one or two instances, the discharge from the ear having been accidentaliy discovered after the fatal disease of the hrain had taken place. I regret to add, that it is too frequent an occurrence for the sympathetic fever attending acute otitis, with its fatal extension to the brain, to be mistaken for typhus, and treated accordingly; the local and primary disease being entirely overlooked, or attention, too late for any useful purpose, being directed to it by accident. The acute tympanitis of scarlet fever generally terminates in extensive chronic disease of the tympanum, which is extremely liable to be excited into the acutc form at some future period, and then may extend to the brain, or in any other direction.

The pathognomonic condition of the mucous membrane of the fauces in scarlet fever extends alike along the Schneiderian membrane and the Eustachian tube ; in the former: in consequence chiefly of the anterior and posterior openings of the nares being so large and free, the morbid secretions readily find their escape, notwithstanding their often immense quantities, the results of epithelial desqua mations, of enormous secretions of mucus, of sero-mucus, of muco-pus, and of pus itself, whether the products of ulceration, or proceeding from the highly turgescent membrane. Bleeding not at all infrequently occurs, sometimes in large quantities, either combined with other matters, or alone. We are not surprised, then, that most serious disease of the nostrils, implicating the spongy and other bones, should follow this frightful calamity : nay, it is rather to be wondered at that the complete recoveries from such extensire miechief are 80 common. I think I may conclude, but I speak under correction, that this scarlatinous inflammation does not so constantly extend along the Eustachian tube as it does into the nares; and, in consequence of the narrower canal of the former, and probably also because the tympanic mucous membrane, being further remored in structure from the external skin, is less predisposed to the eruptive disease than the Schneiderian membrane; but of this circumstance again I speak with diffidence. Notwithstanding this doubtfully expressed opinion, I do not at all hesitate to declare that, in my experience, the extension of scarlet fever is by far the most frequent cause of destructive intlammation of the middle ear; and, including its extension along both conduits-the external auditory canal and the Eustachian tube-I believe it as frequent as, or even more frequent than, all the other exciting causes together, not even excepting measles, which is the next in frequency as a single cause. I beliere the same destructive inflammation takes place in the carity of the tympanum that we recognise so readily upon ocular examination of the nostrils; and to the same extent, with the exception of the amount of cpithelial desquamation, which, in mild cases of scarlet otitis, may be slight enough to be dissolved or suspended in the increased quantity of mucus, and with it be absorbed, or escape by means of the Eustachian tube. From this mildest effect, hardly to be recognised as disease, we mect with every variety of degree, to the complete destruction of the entire aural organ. Thus, muco-tympanitis, scarcely to be distinguished from catarrhal, except by the producing cause, occasioning partial and temporary deafness, readily evidenced in the increased vascularity of the membrana tympani, and readily yielding to treatment. An increased amount of inflammation may give rise to a chronic increased action of the mucous membrane, keeping up a constant accumulation of mucus in the cavity. Then we find a still further effect in the effusion of fibrin, thickening the lining membrane, forming bands across the carity, uniting the ossicles so firmly as to prevent their movements, and sometimes stretching from the membrane in front to the osseous wall behind; and, being organised more or less completely, these bands contract, drawing the membrana inwards. This state of the tympanum cannot well occur, if it can at all, without an accompanying thickening and opacity of the true membrane; and hence very complete deafness often results. Even in such cases of adhesive inflammation of the mucous and submucous tissues, it is not common for the Eustachian tube to be obliterated, although its canal may be contracted. In severe attacks of scarlet fever, the scarlatinous inflammation of the mucous membrane of the tympanum generally proceeds to suppuration, most probably preceded by adhesion, slight and temporary, and succeeded by ulceration, but by no means suspended by it: the rapidity of its progress, the direction of its course, the extent of its destructive results, the severity of its symptoms, its amenability to treatment, must depend upon the peculiarities of the general disease, of the local predispositions, and especially upon the idiosyncrasies of the patient. Most commonly, the membrana tympani, being thinned by the ulcerative process on its inner surface, and not infrequently also upon its outer, suddenly gives way, allowing the matter to freely discharge into the external canal; and usually with relief of pain to the sufferer. Mr. Wilde says, the membrane usually first yields at the lower and fore part; but this statement does not accord with my experience. The situation of the ulcer seems to depend rather upon the local severity of the inflammation, than upon any peculiarity of the particular part of the membrane; and more frequently to be in the upper than in the lower part of the membrane, which may possibly have some connexion with the sinus of the tympanum - with the matter lodging there in larger quantities than elsewhere (for we must not forget that the patient is necessarily in the recumbent posture); and it may have some connexion with the greater tenuity of that part of the membrane.

It is an interesting fact, and one which we should readily 
comecive that the mambrane does not neuslly gire ray at the attachment of the malleus, it being supported by that little bone. I have frequently soen a strip of the membrane remain attached to the entire length of the manubrium of the malleus, each retaining the other in position; the rest of the membrane having been ulcerated away, with the occasional exception of a portion of the margin. Then, again, it is not at all uncommon to meet with a central aperture in the membrane, the rest being entire, though in a state of vascular hypertrophy: the aperture being in the position normally occupied by the manubrium of the bone, I have imagined that the malleus has been displaced, and escaped through the opening, with or without the incus. This circumstance appears to be connected with the natural greater vascularity of that part of the membrane. The ossicula auditus do not always exfoliate when the membrana tympani ulcerates: they may remain altogether in situ, unsupported by the membrane, being attached (perhaps by abnormal adhesion) to each other, and by the head of the malleus to the sinus tympani, and the base of the stapes to the fenestra ovalis; or the malleus may be lost, leaving the incus and stapes in position. But it as frequently occurs, perhaps more so, that the incus becomes detached from both the malleus and stapes, and escapes with the discharge from the tympanum, leaving the other ossicles in their proper positions.

The common effect of extensive ulceration of the membrane upon the bones is the loss of both the malleus and incus, including of course the orbicular, leaving the stapes in situ, though often concealed from view by the granulated mucous membrane; and this condition may exist with very fair hearing. The stapes is also not infrequently removed with the other bones; and if the membrana fenestra oralis be entire, and unconcealed by discased mucous membrane, as I have stated on former occasions elsewhere, hearing may still be enjoyed to a considerable extent; and in one instance I have known it not appreciably defective. The mucous membrane submits to many and great alterations in these unhappy cases; it is generally hypertrophied, and secreting large quantities of pus; it often becomes a pyogenic membrane, the mucous follicles giving place to purulent follicles, always vascular, often spongy, and very frequently fungoid. This state of mucous membrane is the common cause of the long-continued, obstinate, offensive, and dangerous otorrhoa, we so constantly encounter; the matter discharged changing from pure pus to muco-pus, and back again; and sometimes even assuming the appearance of true mucus, or sero-mucus, as the vascular excitement of the diseased mucous membrane may rary. A few vicinious pus-follicles may become more vascular, more nourished than the rest: they consequently sprout forwards beyond the general surface of the membrane into a fungoid granulation, rendering the surface very unequal, and producing a more copious secretion. Several of such granulations coalescing, they project forwards into a prominent fungus; and, pushing through the opening in the membrane, it occupies the meatus, retaining at first its extreme vascularity, still copiously secreting, and easily bleeding; but, as it grows, spreading further and further from its attachment in the cavity, it necessarily becomes less vascular, more transparent, with a few vessels now seen ramifying through it, capable of maintaining its growth; but, incapable of affording secretion, it assumes the character of a polypus. Secretion still goes on in the tympanic cavity, and also from the root of the polypus, where the great vascularity is continued; and thus the otorrhoea, and of ten to a great extent, is continued. During this proceeding, it appears that only occasionally matter escapes through the Eustachian tube; and yet there is rarely a stricture, as is proved by catheterism, by air being readily admitted upon deglutition, and upon forcible expiration with the nose and mouth closed, and often transmitted into and through the outer meatus, and by water flowing into the nose and pharynx upon syringing the external ear. I expect that the granulated mucous membrane lies over the narrowed tympanic opening of the tube, and closes it to ordinary oc- casions ; but that the folds of membrane are separated upan extraordinary occurrences.

Facial paralysis occasionally occurs as a complication with disease of the tympanum ; the inflammation extends through the parietes of the aqueduct of Fallopius, and diseases the portio dura in its track; or the aqueduct may actually be ulcerated, and the nerve either pressed upon, or mixed up with the general disease of the cavity.

These are the conditions of the middle ear most constantly presenting themselves, and which are so very prono to be eusily excited into an acute intlammation, and that upon a comparatively insignificant cause; a happier result of this condition, and not at all an unusual one, is the absorption or ulceration of the entire mucous membrane, leaving the periosteum alone covering the osseous parietes of the cavity; generally, in such cuses, the two externa bones are lost, and not infrequently the stapes also, but all the ossicula may remain, they alike being denuded of their mucous corering. When the bones are remored, the posterior wall of the tympanum is plainly brought into view, the promontory is seen projecting, and upon its surface I think I have often detected the grooves previously occupied by the tympanic plexus of nerves (for I expect they are necessarily removed with the mucous membrane); striking the promontory with a probe gives rise to little or no sensation to the patient, though to an unmistakeable one to the surgeon; the outline of the fenestra rotunda is readily distinguished, its membranc of course remaining concealed; the head of the stapes is brought into view, and if removed, there is seen the margin of the fenestra ovalis, and in one instance I distinctly recognised the nembrane, and in which case the sense of hearing continued surprisingly acute. I rejoice to congratulate the patient upon this condition of the tympanic cavity: deafness, it is true, is the general consequence, but even that defect raries much in degree; the periosteum and the bone, under these circumstances, are so little prone to morbid action, that any further result of disease is very unlikely, and I never anticipate any return of otorrhoca, or extension of disease to other structures.

In some milder and more promising cases, the pus or mucus, or both, of scarlatinous otitis finds its way into the pharynx through the Eustachian tube, as is $\in$ ridenced by the paticnt feeling the somewhat sudden gush, tasting the matter, and by the subsidence more or less of the ear symptoms; he may not, however, always feel or taste the discharge, in consequence of the still morbid condition of the pharynx, fauces, mouth, etc., yet we hare good reason to expect it has thus escaped by the improvement of the ear being too sudden to have occurred from absorption only. This, of course is the happiest mode in which such relief can be afforded, and which is occasionally brought about by a forcible entry of air through the tube as in the effort of coughing, sneezing, blowing the nose, etc., etc. Unfortunately, this species of otitis also spreads with direful effect in other directions, and, as may be supposed, backwards into the mastoid cells; but even there it may occur in the mild form, and subside with the recovery from the general fever. It is, however, excessively liable to terminate in mastoidal abscess greatly aggravating the tympanic affection; and yet sometimes acting as a derivitant to the tympanic disease; here the same conditions of the mucous lining may occur as those that we have already noticed; but the most common consequence is an abscess situated external to the bone between it and the periostcum; the inflammation has extended from the mucous membrane through the bone to the external periosteum, and there engendered an abscess; and this condition certainly takes place, in the great majority of instances, beforc the bone dies or is dying, and if early and properly treated, the bone does not usually exfoliate, the integument is preserved from ulceration, and the case generally does well ; but when neglected, the pus does not quickly find its way to the surface through the fibrous unyielding textures, the bone becomes still further inflamed, and dies before the abscess has pointed, generally terminating in exfoliation, and sometimes forming a com- 
menication between the external ulcer and the cavity of the trmpanum through the mastoidal cells; the extent of exfoliation varies greatly; a fungus not infrequently shoots from the mucous membrane through the external ulcer, altogether occasioning a state of parts very difficult to manage, exceedingly disagreeable and offensive to the patient and his friends, and not free from danger of extending inwards to the brain or to the lateral sinus.

The active disease of the mucous membrane of the tympanum, as is too well known to every practitioner by sad experience, extends its baneful effects to the brain, lighting up infiammation of a character and degree regulated by the peculiar attendant circumstances, and terminating as determined by predispositions natural or recently acquired by the specific fever. Most frequently the disease takes the course of the roof of the tympanum, thus reaching the middle lobe of the cerebrum ; or, if a little more backwards, reaching the posterior lobe, but the inflammation, in $\mathrm{my}$ expérience, usually affects the posterior or anterior part of the brain by extending along the dura mater or the lower surface of the middle lobe of the brain. I have met with an abscess in the middle lobe, and a secondary one, not communicating, in the anterior lobe. The disease also passes through the posterior wall of the tympanum and reaches the cerebellum, and not very infrequently the lateral sinus itself; or it sometimes mounts upwards through or round the margin of the tentorium to the brain proper. I have usually found that the bone is not carious nor necrosed, but inflamed, which inflames the dura mater, which in its turn transmits it to the structure of the brain, and there results an independent abscess, that is, an abscess not communicating with the ear, but surrounded by brain matter; in several instances, I have seen the bone superficially ulcerated under the dura mater, which still remained attached; and in one instance, an abscess in the cerebellum had a small communication with the ulcerated tympanum; in another, the dura mater, covering the petrous portion, formed the floor of the abscess, the other walls being in the middle lobe; and in another, a direct though small communication existed between the carity of the tympanum and the lateral sinus.

The disease of the brain arising from extension from the ear gives rise not infrequently to effusion of serum between the membranes, or into the ventricles, from which, dangerous in the extreme as it is, recovery may take place; likewise, softening of the brain may occur as the result of this character of inflammatiou. I do not remember to have seen or to have heard of hardening of the structure.

Further, acute scarlatinous otitis extends to, and involves in destruction the labyrinth itself ; in several, nay many, instances, I have seen considerable portions, the bulk of the cochlea which had escaped from the outcr meatus of course through the tympanum, and I think I have been able to recognise bits of the scmicircular canals, but they were so broken up that I could not speak positively; from this circumstance I am inclined to the opinion, that the disease finds its way to the cochlea through the fenestra rotunda, more readily than to the semicircular canals, through the fenestra ovalis and the vestibule. It is very possible that the entire portion of the petrous bone containing the organ becomes in such cases diseased, for exfoliation of the bony case takes place to a greater or less degree with that of the labyrinth, but corresponding to the mode of development the cochlea is more readily separated than the rest. I need not add that these desperate cases are necessarily attended by complete deafness, and, in young subjects, entailing consequent dumbness. Scarlet fever becomes the most fruitful source of deaf-dumbness, far more fruitful even than congenital defects, and perhaps more fruitful than all the other causes combined.

Lastly: scarlet fever, like other general diseases of the system, occasionally locates its principal mischief in the brain, with or without disease of other organs, producing inflammation or congestion, and their various consequences in the brain matter or in the membranes; one of the results of serere affection of this kind is deafneas, usually very complete; and when it occurs without disorganiation of the tympanum or its appendages, or with disorganisation too slight to account for the extreme effect, and especially when it presents other signs of cerebral affection, we are fairly lod to attribute it to disease of the brain in associz tion with the ear; and as the acoustic nerve (with the other nerres of sense) may be regarded as an offset or prolongation of the very brain itself, I have supposed that the nerve, either in its expansion in the labrinth, or in its track, or in its attachment to or origin from the brain, remains in a diseased condition, the rest of the brain having recovered from the effects of the scarlatinous inflammation.

We have not had sufficient opportunities of post mortem inspections to decide what this condition is, but I have acted upon the expectation that effusion of serum or of sero-fibrin has taken place into the tissue of the nerve; its structure may be broken up and softened, or be hardened with deposited fibrin more or less organised. In some-instances, I believe the nerve in some part of its course, possibly in its whole length, more likely in its expansion, to be anæsthenic. It is possible that deposit in the nervous tissue may be recovered from, the anæmic or anæsthenic condition is hardly probable. This state becomes a cause of cophosis so complete as to endanger muteism in the young subject.

I subjoin, as briefly as possible, a few cases in illustration, most of which have presented themselves to my notice within the last few days.

CASE I. Miss B. B., aged 22, had scarlet fever in childhood, and had been deaf since. Both membrana tympani extensively removed; a small piece at the upper and anterior part remains, quite white and thick; watch barely heard in contact with the ear; slight discharge. Ossicles certainly removed: this case was remarkably ameliorated by artificial membranes.

CASE II. Eliza Jones, aged 6, had scarlet fever in 1851, now a deaf mute. Mcatus healthy; tympanum only to be seen in a good light; membrana quite gone; no doubt the ossicles are also; the probe strikes against the promontory. Her parents say she never had discharge, which is most likely incorrect; they detected her complete deafness after recovery, but which almost certainly occurred during the fever; now she is in perfect general health, and with good intellect. It is prohable that the auditory nerve, in its expansion in the labyrinth, is also affected with disease.

CASE III. Miss F., aged 40, had scarlet fever, afterwards typhus-fever, when a child. She was very ill with severe pain in the left ear, from which she experienced sudden relief. She has since been deaf in the left ear, with discharge. Membrana tympani ulcerated in its centre; purulent discharge. She can hear an ordinary watch at two or three inches distance. Right ear well.

CASE Iv. Rev. Mr. - aged 69, had scarlet fever in childhood. He has been quite deaf in the right ear since, with a slight discharge; also deaf in the left ear occasionally. Three weeks ago, he became very deaf in the left ear also. Each meatus is so contracted from the long-continued chronic inflammation as to obscure the membrana tympani. Slight otorrhœa; air forced through the right ear upon powerful inflation; quite deaf on the right; on the left, watch heard an inch or two. Both membrana tympani ulcerated, to what extent it is difficult to see.

CASE v. Mrs. G., aged 35, has had discharge and deafness in the left ear from childhood, in consequence of scarlet fever. Left meatus presents at its lower part a palish vegetation, no doubt proceeding from the tympanic mucous membrane. The ulcerated membrana tympani well seen beyond the growth; free discharge: quite deaf; right ear well.

CaSE vi. A young woman has her left meatus quite filled with a very vascular polypus, bleeding upon the ordinary examination; copious otorrhoca; the left side of the face is completely paralysed; health good; it is impossible to 800 the membrana tympani; but I am sure it is entirely ulcerated away. She states that she has been deaf, and had discharge from that ear since childhood, but does not rocol- 
leet the canes, nox the antecedenta. I have no doubt that to the cufiened from ecarlet foror.

C10 vII. Master B. had scarlet ferer when a child, in wich he nearly died from ear and brain disease. Some months after recovery, I saw him, then perfectly deaf. The membrana tympani were both removed by ulceration, as well as the ossicula. A portion of cochlea was likewise shown me, which had escaped from the ear-copious discharge from a spongy mucous membrane. The membrane charge improved; otorrhoea lessened, and at length removed. He gradually lost his speech, and is now being educated as 2 deaf-dumb.

Case virr. A physician writes me as follows:-" My two children, aged respectively 11 and 13 , were six weeks past attacked with scarlatina anginosa, very intense and protracted; the thront affection accompanied with severe inflammation of the mucous lining of the mouth and nostrils, and spreading to the nasal ducts, and affecting the eyes. Of course the Eustachian tubes partook, and complete deafness in both cases ensued. The suppuration from the ear has been considerable and fotid, but not haring at all the smell of decaying bone; and I have accurately marked the discharge, without being enabled to detect more than a puriform discharge, acrid enough to excoriate the external ear and cheek, but unaccompanied by blood or sanies. The statement of one will serve for both, as no deviation is perceptible." I found in hoth ears of the boy that the membrana tympani was entirely removed. The malleus remained in silu, entirely stripped of its coverings, yet attached to the granulations of the mucous lining of the cavity. Otorrhoea considerable. The speech was gradually being lost; and I believe he is being taught as a deaf-mute.

The case of the sister was as nearly similar as could be. In these cases, I think there can exist little doubt that the labyrinth or the nerve in its track must be affected.

CASE X. Joseph Seamons, a.ged 9, had scarlet fever very dangerously four years ago, and has had discharge from the right ear ever since. The membrana tympani is extensively ulcerated. An abscess formed over the right mastoid process, which was insufficiently opened. A fistulous ulcer communicates between this opening and the posterior surface of the auditory meatus, from which a vascular fungus projects into the auditory canal.

CASE xI. Louisa Eames, aged 17, had scarlet-fever seven or eight years ago. A large polypus grows from the mucous lining of the tympanic cavity of each ear, and completely occupies the meatus.

Case xir. Miss M. J., aged 15, has been deaf ten or eleven years, in consequence of severe scarlet fever, which endangered her life. The deafness gradually increased, accompanied with considerable discharge of a fotid matter, of variable consistence, with formerly occasional bleeding; discharge and bleeding likewise from the nose. The general health now tolerable. The membranæ tympanorum removed by ulceration, except a small portion at the upper part. The mallei and inci both lost, and possibly also the stapedes; mucous membrane hypertrophied; meatus moderately healthy, though secreting a thin fluid; tonsils and fauces healthy.

2, Harley Street, Cavendish Square.

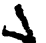

\section{NOTES AND OBSERVATIONS ON SOME OF THE MORE REMEDIABLE CASES OF DEAFNESS.}

By C. HOLTHOUSE, F.R.C.S.E., Assistant-Surgeon to the Westminster Hospital, and Lecturer on Surgical Anatomy in its Medical School.

ro. 1 .

“Ir aural diseases were as attentively studied in these kingdoms as ophthalmic or obstetric affections, then would the lengthened description of cases be unnecessary; but where do wo find throughout the whole circle of our peri- odical litorature half a dozen well observed and sccuratals noted cases of disease of the ear in a trelvemonth 1 Faithful observation and clinical records of disease are now mare ful observation and than in any other branch of medical ecience." Fully concurring in the opinion thus expresed science. by one of the ablest aurists of the present day (Mr. Wildo), trusting that they may in some measure supply this want. They are plain and faithful records of what I actually saw and observed; nothing is stated on mere conjecture; when I have fairly scen a part, I have described as accurately as possible its appearance; if an unsatisfactory view was potained, I hare stated so in the notes. Every case, therefore, as far as it goes, may be relied on for accuracy; the truth has always been told, though possibly not the whole truth, because the necessary examinations to arrive at it, either could not be made, or, having been made, the information sought for could not in every instance even then be obtained.

It may be thought by some that the researches of Mr. Toynbee in the department of aural surgery have made us so fully acquainted with the pathology of diseases of the ear, that further illustrations are unnecessary.

Without, however, any disparagement to this distinguished aurist, the value of whose labours no one can appreciate more fully than myself, I must observe, that hitherto they have been confined almost exclusively to an elucidation of the morbid changes found in the ear after death; while the symptoms which these changes gave rise to during life, the exact amount of deafness which this or that lesion occasioned, we are left in ignorance of. The association of the symptoms with the lesions is, therefore, still a desideratum, which it is hoped this contribution may furnish. In many of the cases reported, there will be found the identical changes in the organ of hearing, which Mr. Toynbee's dissections have revealed; while data are supplied relative to the amount of improvement of which the diseased organ is susceptible.

CASE I. Acute Inflannmation of the Membrana Tympani of both Ears brought on by Cold; Great Deafness, Severe Pain, Loud Tinnitus, followed on the second day by discharge; Right Membrana Tympani covered with Florid Granulations; Left Ecchymosed; Eustachian. Tube of the Left Lar obstructed; Treatment by Leeches, Blisters, Aperients, and afterwards an Astringent Lotion; Hearing restored. John Bradford, aged 46 years (cook), applied to me on the 14th July, for relief from a purulent discharge affecting both ears, and accompanied with pain, a high decree of deafness, and a constant buzzing and ticking tinnitus. On washing away the discharge, by means of a syringe, the membrana tympani of the left ear was less lucent than in the healthy state, but was otherwis? not remarkable; the membrana tympani of the right ear could remarkable; the membrana tympani of the right ear could to detach with the syringe; on gently touching it, however, with a probe, great pain was excited.

History. Eight days ago he caught cold, which, as he said, "stopped up his ears". This was soon followed by severe pain in both ears, accompanied by a humming and blowing, and various odd sounds. The pain continued to increase in severity, when it was considerably relieved by a discharge, which broke forth from both ears on the second day of the pain, just after the application of some leeches; the buzzing and deafness, however, remained undiminished. When the tinnitus first came on, and for some time afterwards, he could not dispossess himself of the idea that the noises proceeded from without; and the delusion was so me see whence they proceeded. He said that he had enjoyed good health; but thirty years ago he had a similar attack, which was got rid of without impairment to hearing.

Hirudines vi, pone aures sing. statim applic.

Habeat haust. cathart. omni mane.

July 15th. The leech-bites bled freely, and the patient was much relieved. He felt lighter in the head, and slept better last night than he had done since the attack. 